\title{
Childhood maltreatment and characteristics of adult depression: meta-analysis
}

\author{
Janna Nelson, Anne Klumparendt, Philipp Doebler and Thomas Ehring
}

\section{Background}

Childhood maltreatment has been discussed as a risk factor for the development and maintenance of depression.

\section{Aims}

To examine the relationship between childhood maltreatment and adult depression with regard to depression incidence, severity, age at onset, course of illness and treatment response.

\section{Method}

We conducted meta-analyses of original articles reporting an association between childhood maltreatment and depression outcomes in adult populations.

\section{Results}

In total, 184 studies met inclusion criteria. Nearly half of patients with depression reported a history of childhood maltreatment. Maltreated individuals were $2.66(95 \% \mathrm{Cl} 2.38$ $2.98)$ to $3.73(95 \% \mathrm{Cl} 2.88-4.83)$ times more likely to develop depression in adulthood, had an earlier depression onset and were twice as likely to develop chronic or treatment-resistant depression. Depression severity was most prominently linked to childhood emotional maltreatment.

\section{Conclusions}

Childhood maltreatment, especially emotional abuse and neglect, represents a risk factor for severe, early-onset, treatment-resistant depression with a chronic course.

\section{Declaration of interest}

None.

\section{Copyright and usage}

(c) The Royal College of Psychiatrists 2017.
Major depression is one of the most prevalent psychiatric disorders worldwide with most lifetime prevalence estimates ranging between 8 and $12 \%{ }^{1}$ The World Health Organization has found it to be a leading cause of disability-adjusted life-years. ${ }^{2}$ However, depression is not a homogeneous disorder; instead, depression severity, symptom patterns and age at onset vary considerably between individuals. ${ }^{3-5}$ Importantly, this heterogeneity has been found to influence the course of illness and response to treatment. ${ }^{6-8}$ It therefore appears essential to identify variables that explain this variance in order to improve our understanding of the phenomenology, aetiology and treatment of depressive disorders. Recently, childhood maltreatment has been discussed as an important factor influencing not only the incidence of depressive disorder but also its characteristics. Several studies have reported childhood maltreatment to be related to a greater depression severity as well as an earlier onset of depression. ${ }^{9-11}$ In addition, a recent meta-analysis revealed individuals with a history of childhood maltreatment to be more than twice as likely to develop recurrent and persistent depressive episodes and about 1.5 times as likely to be non-responders to depression treatment, be it psychotherapy, pharmacotherapy or combined treatment. ${ }^{12}$ Importantly, there is evidence of differential effects of childhood maltreatment subtypes such as emotional, sexual or physical abuse. Summarising 124 studies, Norman et $\mathrm{al}^{13}$ showed that emotional abuse increases the risk of depression by an odds ratio of 3.06, whereas physical abuse increases the risk of depression by an odds ratio of 1.5, merely half that of emotional abuse. Similarly, Spertus et $\mathrm{al}^{14}$ found childhood emotional maltreatment to be more closely related to depression severity than sexual or physical abuse. Childhood emotional abuse and neglect predicted depressive symptomatology even when controlling for physical and sexual abuse. ${ }^{14}$

Following these results, it appears inadequate to regard childhood maltreatment as a unitary phenomenon when considering its effects on depressive disorders. Instead, childhood maltreatment subtypes need to be considered separately.

The aims of the current meta-analysis were twofold. On the one hand, we aimed to provide an update of earlier meta-analyses investigating the effect of childhood maltreatment on characteristics of adult depression. Specifically, the increase in risk of depression in individuals with a history of childhood maltreatment was estimated as a function of different childhood maltreatment types. Furthermore, the influence of childhood maltreatment on course of illness and treatment response was investigated. Our meta-analysis, additionally, included three research questions that, to our knowledge, have not been investigated before using a meta-analytic approach. First, we sought to quantify the percentage of patients with depression who had a history of childhood maltreatment to gain an understanding of the prevalence of each childhood maltreatment type as a risk factor in individuals who have depression. Second, the correlation between childhood maltreatment severity and depression severity was analysed to test a possible dose-response relationship. Finally, the influence of childhood maltreatment on age at onset of depressive disorders was analysed. In summary, the current meta-analysis provides not only a comprehensive update regarding the influence of childhood maltreatment on depression incidence, course of illness and treatment response but extends earlier findings by examining depression severity, age at onset and prevalence of childhood maltreatment types in individuals with depression.

\section{Method}

\section{Inclusion criteria}

We identified studies satisfying the following criteria: (a) study includes an assessment of childhood maltreatment defined as sexual, physical or emotional abuse, and/or physical or emotional neglect up to age 18; (b) study includes an assessment of a diagnosis of depressive disorder or severity of depressive symptoms; (c) study includes an adult population-based or clinical sample; (d) study reports data on at least one of the following issues: (i) prevalence of childhood maltreatment in a sample with depression, (ii) risk of depression, chronic course of depression or negative treatment outcome in maltreated and 
non-maltreated samples, (iii) correlations between childhood maltreatment severity and depression severity, (iv) age at depression onset in maltreated $v$. non-maltreated samples.

\section{Search strategy}

Three electronic databases (MEDLINE, PsycINFO, PILOTS) were searched up to 21 November 2013 using abstract or title search to identify fully published, peer-reviewed journal articles in English, French or German (for search terms see Fig. 1). In addition, reference lists of earlier meta-analyses ${ }^{12,13,15,16}$ and reviews ${ }^{17-21}$ were searched using the same criteria as above.

\section{Data extraction}

Data extraction from eligible articles was performed independently by two coders using a standardised data extraction sheet and coding manual. Disagreement was resolved by consensus. Main coding variables included information on study group (i.e. sample type, sample $n$, percentage of women in sample, mean age of sample), childhood maltreatment (i.e. childhood maltreatment type, definition of childhood maltreatment, childhood maltreatment measure) and depression (i.e. type of depression measure) as well as study design. For research questions requiring dichotomous measures of childhood maltreatment, 'at least moderate childhood maltreatment' was compared with 'no childhood maltreatment', and for those requiring dichotomous measures of depression, 'major depression' was compared with 'no depression' whenever possible. For chronicity of depression, definitions of the respective study were used. With regard to treatment response, a cut-off score in 'percentage improved' was preferred to an absolute cutoff-score to define positive response to treatment whenever both were reported.

\section{Keywords:}

chronic*/duration, severe/severity/symptom level*, treat*/therap* outcome/respon*, subtyp*/symptom*/cluster

AND

depress*/mood disorder

AND

trauma*, child* abus*, physical* abus*/sexual* abus*/emotional* abus* early interpersonal trauma, child* maltreat*

NOT brain injury

\section{$\nabla$}

Studies identified through literature search $(n=10482)$

- PsycINFO: $n=3241$

- MEDLINE: $n=3620$

- PILOTS: $n=3621$

Studies identified for abstract review $(n=6247)$

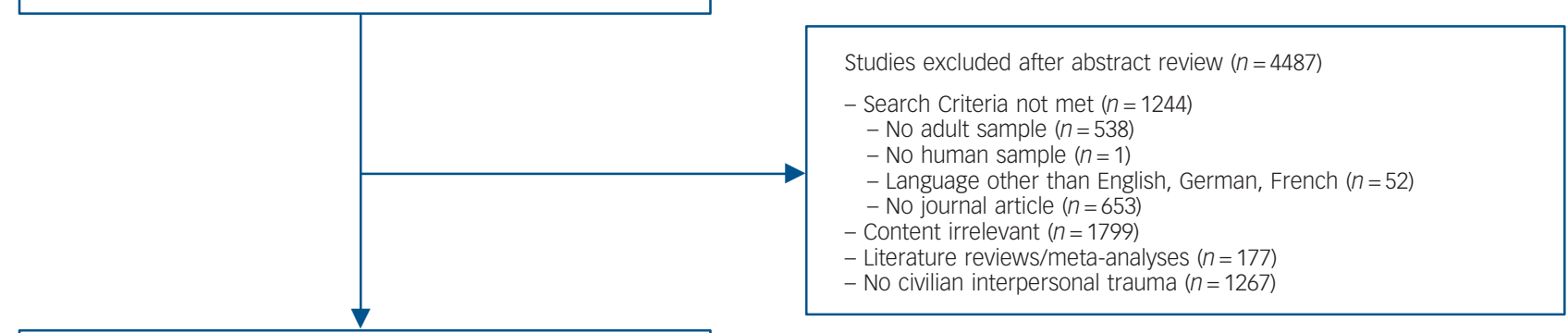

Studies identified for full-text review $(n=1760)$

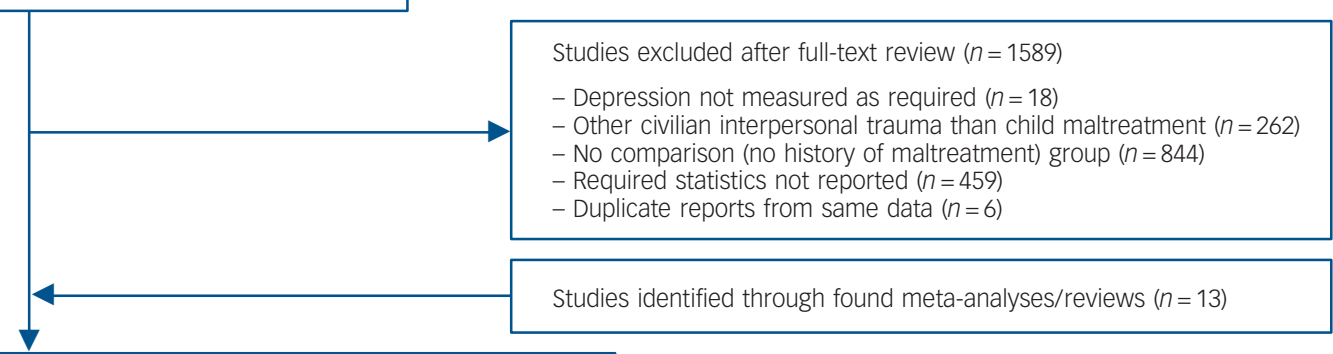

Studies included in meta-analysis $(n=184)$

- Prevalence of maltreatment types in depression $(n=118)$

- Risk of depressive disorders in individuals who were maltreated $(n=83)$

- Correlation between maltreatment and depression severity $(n=52)$

- Risk of chronic depression in individuals who were maltreated $(n=10)$

- Age of depression onset in individuals who were maltreated $(n=10)$

- Response to treatment in individuals with depression who were maltreated $(n=5)$ 


\section{Statistical analyses}

Software

Analyses using only one end-point per study were performed using Comprehensive Meta Analysis (CMA, version 2.2; Biostat). This was the case for research questions 1-3 since there were enough studies to run separate analyses for each subtype of maltreatment. For research questions $4-6$, there were not enough studies to run separate analyses by maltreatment type. Therefore, all study end-points were combined into one analysis per research question. This combination leads to a higher statistical power by increasing the number of included effect sizes but its validity is threatened by non-independence of effect sizes. In order to minimise bias, robust variance estimation with small sample adjustments $(\mathrm{RVE})^{22,23}$ was implemented via the robumeta package in $R^{24}$ to account for unknown correlations among these non-independent samples whenever multiple end-points per study were used.

\section{Effect sizes}

For statistical analyses of the prevalence of childhood maltreatment in individuals with depression, event rates were converted to logit event rates for analyses. Logit-transformation was used to dissolve restricted range of event rates and assume normal distribution, but results were back-transformed into percentage values to allow a more intuitive interpretation. For analyses of risks (i.e. risk of depressive disorder, chronic depression and negative treatment outcome), extracted data were converted to log odds ratios for the same reasons as using logit event rates instead of event rates. Whenever possible, log odds ratios were computed directly from raw data $(2 \times 2$ tables $)$ to obviate systematic differences based on varying formulas for calculation of effect sizes. Again, to ease the interpretation results were reconverted to odds ratios reflecting the probability of unfavourable outcomes. Partial odds ratios controlled for other variables were excluded to alleviate contortion of data. To aggregate correlations between severity of childhood maltreatment and severity of adult depression, Pearson's or Spearman's correlations were converted to Fisher's $z$ and later back-transformed into correlations for interpretation. To test the hypothesis that individuals with a childhood maltreatment history have an earlier onset of depression, raw mean differences in age at onset between maltreated and non-maltreated individuals were calculated.

\section{Heterogeneity}

It seems inappropriate to assume one single true effect to underlie studies using different populations and assessment methods. We therefore took a random-effects model as a basis of our analyses. $Q$, Higgin's $I^{2}$ and $\tau$ are reported as measures of heterogeneity. ${ }^{25}$

\section{Sensitivity analyses}

Whenever studies with multiple end-points are combined into a single analysis without knowledge of the variance-covariance matrix of end-points within the respective study, the within-study correlation of end-points $\rho$ needs to be imputed. As this method is potentially error-prone, sensitivity analyses implementing different values for $\rho$ were performed.

\section{Publication bias}

Effects of publication bias may compromise the validity of metaanalytic results. ${ }^{26}$ Evidence of publication bias was investigated visually by use of funnel plots and statistically via Egger's test ${ }^{27,28}$ and Duval \& Tweedie's trim and fill analysis. ${ }^{29}$ Whenever effect sizes were odds ratios, Peter's test ${ }^{30}$ was implemented. For metaanalyses using an RVE approach, a meta-regression predicting the combined effect through the inverse of sample size was used as an equivalent of Peter's test.

\section{Moderators}

Effects of categorical variables (i.e. sample type, type of depression measure, childhood maltreatment measure) on meta-analytic results were assessed using subgroup analyses. Effects of dimensional variables (i.e. mean age of sample, mean percentage of women in sample, quality of assessment) were investigated using random-effects meta-regressions. For quality of assessment, scores ranging from 0 to 2 were given for validity of childhood maltreatment and depression measures, respectively. Scores were then added producing ad hoc quality of assessment scores between 0 and 4. Since these analyses of moderators are explorative in nature, $P$-values were corrected following Bonferroni-Holm. ${ }^{31}$

\section{Differential effects of maltreatment types}

For research questions 1-3, we compared the calculated effect sizes across different types of childhood maltreatment. Univariate approaches were not feasible in these analyses because childhood maltreatment types are correlated (i.e. individuals who have experienced sexual abuse are more likely to also have experienced emotional abuse than individuals unaffected by childhood maltreatment). We therefore implemented the RVE method also used for research questions 4-6. In addition to modelling correlated outcomes, RVE allows regressing them on covariates. We used an extension of the RVE method by Tipton \& Pustejovsky ${ }^{32}$ to compare the effects of different types of maltreatment with the grand mean effect (averaged over all maltreatment types) for research questions 1-3. As different types of childhood maltreatment are correlated, we calculated $F$-tests that account for these intercorrelations by correcting the associated degrees of freedom using a Satterthwaite approximation. $P$-values were corrected following Bonferroni-Holm. ${ }^{31}$

\section{Results}

Our initial literature search yielded 10482 hits. Of these, 6247 were identified for abstract review, and 1760 for full-text review. Finally, 184 studies were included in meta-analyses (see Fig. 1 for full selection procedure and online supplement DS1 for a list of studies).

\section{Analysis 1: prevalence of childhood maltreatment types in adults with depression}

The prevalence of different types of childhood maltreatment in adults with depression was estimated using 118 studies with a total of 255 effect sizes. Random-effects models indicate prevalence estimates from $25.27 \%$ for childhood sexual abuse to $43.20 \%$ for childhood emotional neglect. In total, $45.59 \%$ of individuals with depression reported any childhood maltreatment and $19.13 \%$ report more than one form of childhood maltreatment (Table 1, see online Figs DS1-7 for forest plots).

\section{Analysis 2: risk of adult depression in individuals with a childhood maltreatment history}

The risk of adult depression in individuals with childhood maltreatment compared with those without childhood maltreatment was tested using 83 studies with a total of 175 effect sizes. Randomeffects models indicate that all childhood maltreatment types 


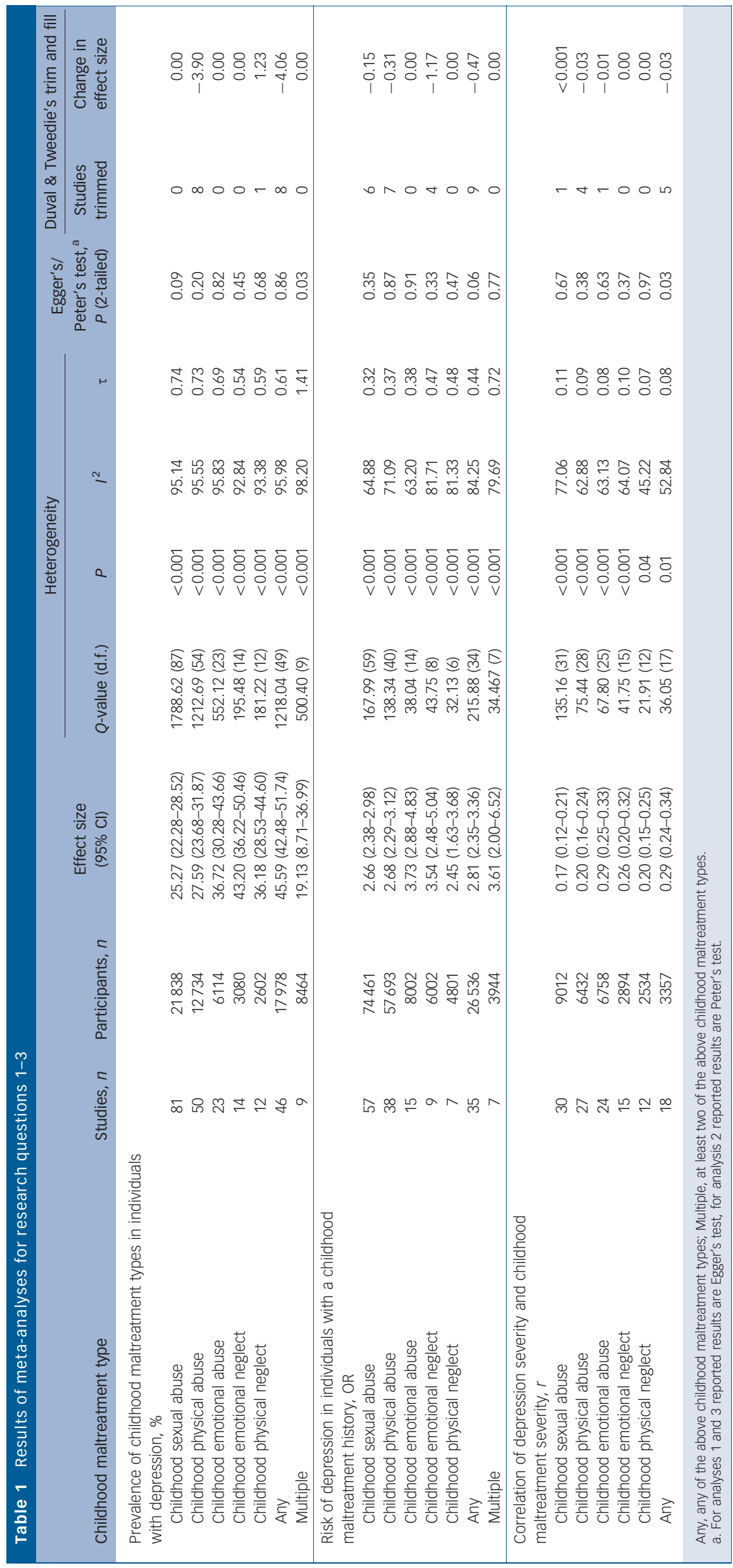


significantly increase the chance of depression in adulthood (Table 1). Regarding single childhood maltreatment types, the smallest increase was observed for childhood physical neglect (odds ratio $(\mathrm{OR})=2.45)$, the highest increase for childhood emotional abuse $(\mathrm{OR}=3.73)$. Any childhood maltreatment increases risk of depression with an odds ratio of 2.81, multiple forms of maltreatment with an odds ratio of 3.61. (See online Figs DS8-14 for forest plots.)

\section{Analysis 3: correlation between childhood maltreatment severity and depression severity}

Correlations of depression severity and childhood maltreatment severity were analysed using 52 studies with a total of 134 effect sizes. Random-effects models showed all childhood maltreatment types to correlate with depression severity on a statistically significant level. Correlations ranged from $r=0.17$ for childhood sexual abuse to $r=0.29$ for childhood emotional abuse (Table 1, see online Figs DS15-20 for forest plots).

\section{Analysis 4: age at depression onset in individuals with a childhood maltreatment history}

The mean age at onset of depression was compared between individuals with a history of childhood maltreatment and those without such a history. Raw mean differences were computed from ten studies with a total of 2683 participants. As there were not enough studies to compute effect sizes for different childhood maltreatment types separately, all 14 study end-points were combined into one analysis using RVE. Random-effects analysis showed mean depression onset to occur roughly 4 years earlier (raw mean difference 4.39 years, s.e. $=0.96$ ) in individuals with a history of childhood maltreatment compared with individuals without such a history. More specifically, maltreated individuals showed a mean age of 23 years at first depression onset, compared with a mean age of 27.1 at first depression onset in nonmaltreated individuals. Note that the difference between these means is not identical to the pooled raw mean difference of 4.39 , since the quantities were computed in three separate RVE meta-analyses. (See online Fig. DS21 for forest plot.)

\section{Analysis 5: risk of chronic depression in individuals with a childhood maltreatment history}

The risk of a chronic course in individuals with both depression and a childhood maltreatment history compared with those without such a history was tested using 11 studies with 14 end-points including 6194 participants. Using RVE, random-effects analysis showed that depressive disorder is twice as likely $(\mathrm{OR}=2.05$, 95\% CI 1.40-3.00) to take a chronic course in individuals with a history of childhood maltreatment. (See online Fig. DS22 for forest plot.)

\section{Analysis 6: risk of non-response to depression treatment in individuals with a childhood maltreatment history}

The risk of non-response to depression treatment in individuals with a childhood maltreatment history was analysed using five studies with a total of 1229 participants. Combining the 13 end-points using RVE, random-effects meta-analysis showed that individuals with a history of childhood maltreatment are more likely $(\mathrm{OR}=1.90$, 95\% CI 1.05-3.46) not to respond to depression treatment than individuals without such a history. (See online Fig. DS23 for forest plot.)

\section{Heterogeneity}

For analyses $1-3$, heterogeneity was tested using a $Q$-test. There was evidence for heterogeneity in all 20 subanalyses, with Qs ranging from 21.91 to 1788.62 , all $P<0.05$, thus supporting the theory-based decision of implementing random-effects models (Table 1). Analyses 4-6 included non-independent effect sizes. Therefore, $\tau$ was calculated as a measure of heterogeneity. For age at depression onset $\tau$ was 2.07, which corresponds to a $Q_{E}$ of 23.56 (d.f. $=9.01, P=0.01$ ). For risk of chronic depression $\tau$ was 0.41 , which corresponds to a $Q_{\mathrm{E}}$ of 31.81 (d.f. $=10.01$, $P<0.001)$. These values indicate heterogeneity and support the use of random-effects models. Solely for risk of non-response to treatment the $Q_{\mathrm{E}}$ statistic of 7.18 (d.f.=5.06) did not confirm the a priori choice of a random-effects model $(P=0.21)$, but inspection of the forest plot showed that the large study of Peyrot et $a l^{33}$ found a lower odds ratio of 1.15 (95\% CI 1.08-1.22) than the majority of smaller studies. Thus, a random-effects RVE analysis was nevertheless reported. Beyond supporting the decision of implementing random-effects models, these high levels of heterogeneity need to be considered when interpreting reported meta-analytic results.

\section{Publication bias}

For analyses 1 and 3, evidence of publication bias was assessed via funnel plot, Egger's test and Duval \& Tweedie's trim and fill analysis. Egger's regression was significant for 2 of 13 subanalyses. Duval \& Tweedie's analysis showed evidence of publication bias for 7 of 13 subanalyses. The changes in effect sizes after study imputation, however, were only minor and did not influence the overall results (Table 1). For analysis 2, Peter's test showed no evidence of publication bias for any of the seven subanalyses. Duval \& Tweedie's trim and fill analyses suggested publication bias in four of seven subanalyses. As with analyses 1 and 3, however, changes in effect sizes after study imputation were only minor (Table 1). For analyses 4-6, an RVE equivalent of Peter's test was implemented. Results showed no evidence of publication bias for any of these analyses.

\section{Sensitivity analyses}

As explained above, $\rho$ had to be imputed in analyses 4-6. To check for possible bias induced by this method, sensitivity analyses were performed implementing different values for $\rho$. These analyses showed no significant changes in effect estimates as $\rho$ was varied.

\section{Analyses of moderators}

To test possible explanations of observed heterogeneity, three meta-regressions and three subgroup analyses were performed. Results of all analyses can be found in Table 2. A first set of meta-regressions used percentage of women as a predictor for respective effect sizes. Only 1 out of 23 meta-regressions was significant: percentage of women in the sample influenced the prevalence estimate of childhood sexual abuse in individuals with depression, in that samples with a higher portion of women showed higher prevalence of childhood sexual abuse.

A second set of meta-regressions used mean age of sample as a predictor for respective effect sizes. In total, 7 out of 22 metaregressions were significant. With the exception of childhood physical abuse and multiple forms of maltreatment, all childhood maltreatment forms were reported less frequently, the older the sample. Mean age of sample also influenced the odds ratio for depression: in the analysis of 'any maltreatment form', older samples showed higher odds ratios for depression than younger 


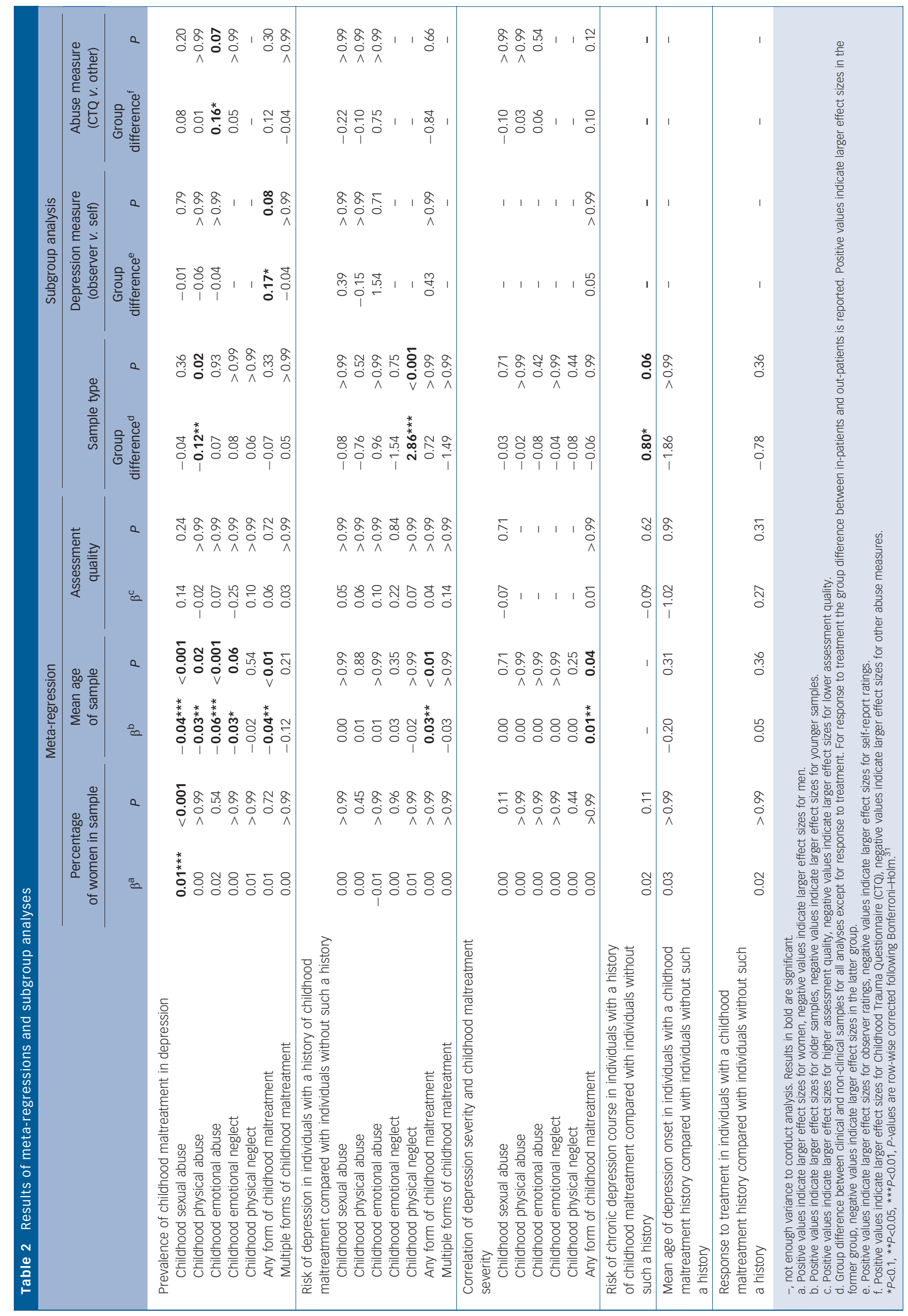


samples. A third set of meta-regressions tested the effects of quality of assessment on effect sizes. Assessment quality of depression and maltreatment measures did not influence any of the reported results.

We conducted a first set of subgroup analyses to compare results across sample types. When comparing clinical and nonclinical samples, 3 out of 22 analyses showed significant differences: clinical samples reported childhood physical abuse less frequently than non-clinical samples. Childhood physical neglect led to a higher increase in risk of depression in clinical samples, as did overall childhood maltreatment in the risk of chronic depression. In a second set of subgroup analyses, we compared results between observer-rated and self-rated depression measures. Only for the prevalence of any form of childhood maltreatment did the results between studies implementing observer ratings of depression differ significantly from those implementing selfratings in that the former approach yields slightly higher prevalence estimates. Finally, a third set of subgroup analyses consisted of comparisons between the results of studies relying on the Childhood Trauma Questionnaire (CTQ) as the most prominently used measure of childhood maltreatment and studies implementing other methods of assessment. Solely the prevalence estimate of childhood emotional abuse appears to be influenced by the assessment method of childhood maltreatment, in that childhood emotional abuse was reported slightly more frequently in studies implementing the CTQ than studies using other assessment methods. Beyond the results reported in Table 2, the effect of childhood maltreatment on treatment response was compared between psychotherapy and pharmacotherapy. Results showed no significant difference in odds ratios between the two treatment approaches $(\beta=0.28, P=0.78)$.

\section{Differential effects of maltreatment types}

To test for differential effects of separate childhood maltreatment forms, we compared effect sizes of individual types of childhood maltreatment to the grand mean (averaged across all childhood maltreatment types). Results of these comparisons can be found in Table 3. Statistically significant differences of individual maltreatment types to the grand mean are emphasised in bold. Concerning prevalence of childhood maltreatment in depression, childhood emotional neglect or any form of childhood maltreatment appears to be more common than the grand mean of the prevalences. With regard to the risk of depression, there are no significant differences between individual types of childhood maltreatment. Finally, regarding the correlation between maltreatment and depression severity, childhood emotional abuse appears to be more closely related to depression severity compared with the overall average.

\section{Discussion}

In accordance with earlier meta-analyses ${ }^{12,13,15,16}$ the current study found that childhood maltreatment elevated the risk of depressive disorders, the chronic course of illness and non-response to treatment. In addition, childhood maltreatment led to an earlier onset of depression. Furthermore, a dose-response relationship between severity of childhood maltreatment and depressive symptom level was found on a small to medium scale. ${ }^{34}$ These results gain additional importance as nearly $46 \%$ of patients with depression reported a history of childhood maltreatment.

Whenever data allowed for differential consideration of separate types of childhood maltreatment, emotional abuse or neglect appeared to be of particular importance. For prevalence of childhood maltreatment in depression, risk of depression as well as correlations between childhood maltreatment and depression severity, a consistent trend of larger effect sizes for emotional abuse and neglect was found. When looking at statistically significant differences only, emotional neglect was the most commonly reported form of childhood maltreatment in individuals with depression, and emotional abuse was shown to be the most closely related to depression severity.

\begin{tabular}{|c|c|c|c|c|c|}
\hline Childhood maltreatment type & Effect size & $F$ & d.f. $\cdot 1$ & d.f.2 & $P$ \\
\hline \multicolumn{6}{|c|}{$\begin{array}{l}\text { Differences in prevalence (\%) of childhood maltreatment types } \\
\text { in individuals with depression (grand mean: } 31.90 \% \text { ) }\end{array}$} \\
\hline Childhood sexual abuse & 24.78 & 5.68 & 1 & 56.37 & 0.10 \\
\hline Childhood physical abuse & 27.45 & 2.41 & 1 & 53.41 & 0.42 \\
\hline Childhood emotional abuse & 37.02 & 0.68 & 1 & 22.31 & 0.84 \\
\hline Childhood emotional neglect & 43.86 & 9.51 & 1 & 13.78 & $0.05^{*}$ \\
\hline Childhood physical neglect & 33.60 & 0.13 & 1 & 12.78 & 0.84 \\
\hline Any form of childhood maltreatment & 46.48 & 20.15 & 1 & 58.09 & $<0.001 * * *$ \\
\hline Multiple forms of childhood maltreatment & 16.48 & 3.26 & 1 & 8.78 & 0.42 \\
\hline \multicolumn{6}{|c|}{$\begin{array}{l}\text { Differences in risk of depression }(\mathrm{OR}) \text { in individuals with a childhood } \\
\text { maltreatment history (grand mean } \mathrm{OR}=3.01 \text { ) }\end{array}$} \\
\hline Childhood sexual abuse & 2.75 & 1.13 & 1 & 22.63 & $>0.99$ \\
\hline Childhood physical abuse & 2.79 & 0.74 & 1 & 26.52 & $>0.99$ \\
\hline Childhood emotional abuse & 3.82 & 4.53 & 1 & 12.30 & 0.37 \\
\hline Childhood emotional neglect & 3.24 & 0.28 & 1 & 7.33 & $>0.99$ \\
\hline Childhood physical neglect & 2.31 & 2.93 & 1 & 5.44 & 0.72 \\
\hline Any form of childhood maltreatment & 2.49 & 2.66 & 1 & 23.47 & 0.70 \\
\hline Multiple forms of childhood maltreatment & 4.13 & 1.38 & 1 & 6.26 & $>0.99$ \\
\hline \multicolumn{6}{|c|}{$\begin{array}{l}\text { Differences in correlations }(r) \text { of depression severity and childhood } \\
\text { maltreatment severity (grand mean } r=0.25 \text { ) }\end{array}$} \\
\hline Childhood sexual abuse & 0.19 & 4.20 & 1 & 28.53 & 0.25 \\
\hline Childhood physical abuse & 0.22 & 1.72 & 1 & 25.63 & 0.40 \\
\hline Childhood emotional abuse & 0.30 & 16.12 & 1 & 20.95 & $<0.01 * * *$ \\
\hline Childhood emotional neglect & 0.27 & 0.478 & 1 & 14.71 & 0.50 \\
\hline Childhood physical neglect & 0.20 & 4.46 & 1 & 11.92 & 0.25 \\
\hline Any form of childhood maltreatment & 0.28 & 2.79 & 1 & 26.06 & 0.32 \\
\hline
\end{tabular}




\section{Significance of our findings and implications}

Comparing different types of childhood maltreatment is interesting and challenging at the same time. Although statistical concerns can be dealt with using RVE analyses and associated corrections, the fact remains that childhood maltreatment types are intercorrelated and this needs to be considered when interpreting results. We are nonetheless convinced that knowledge about differential effects of individual types of childhood maltreatment is relevant. It highlights, for instance, the importance of emotional maltreatment, which does not classify for the DSM definition of trauma (as opposed to physical or sexual abuse). We also hope that our results inspire continuing research into moderators between different childhood maltreatment types and respective sequelae. Regarding practical implications, clinicians, child-care workers and politicians may pay closer attention to forms of maltreatment that are more easily overlooked (i.e. emotional abuse and neglect) when knowledgeable about their consequences.

In sum, childhood maltreatment, especially in the form of emotional abuse or neglect, represents a relevant risk factor in the development of severe, early-onset, chronic and treatmentresistant depression. Our study extends the findings of earlier meta-analyses by including primary studies in a more comprehensive way, assessing a larger number of depression characteristics as outcome variables (such as age at onset, depression severity), and statistically controlling for non-independence of multiple end-points taken from the same study.

\section{Limitations}

Several limitations must be taken into account when interpreting these results, however. First, our results show high levels of heterogeneity indicating much variance in reported findings. This heterogeneity is not surprising as our study concentrates on childhood maltreatment as only one - quite distal - risk factor for depression among many others, but this must be considered when interpreting our results. Even significant pooled effects do not exclude the possibility that in some settings the effect vanishes entirely. Reported meta-regressions and subgroup analyses were aimed at explaining some extent of this heterogeneity, but were limited by information provided by primary studies. Variables such as comorbid disorders, time and length of maltreatment, or perpetrator, which could explain some of the variance, were reported in very few primary studies.

Another issue arises with respect to statistical control. Many primary studies control their effects for age, gender or other sociodemographic variables to account for the potential influence of these parameters. The high heterogeneity in the way primary study researchers adjust analyses, however, creates difficulties at the level of meta-analysis. It becomes extremely difficult to meta-analyse adjusted effect sizes and it calls into question the comparability of the resulting adjusted effects. For these reasons, we have included only unadjusted effect sizes in our metaanalyses. Although leading to a clearer statistical analysis, this decision also has downsides that need to be considered. Reported results are not adjusted for potential confounding covariates. Variables that are correlated both with childhood maltreatment and adult depression may therefore bias our results. Our metaregressions and subgroup analyses of the prevalence of childhood maltreatment in depression provide some information as to which variables could be of interest in this regard. Childhood sexual abuse, for example, appears to be more common in samples with a high percentage of women. As female gender is also known to be correlated with depression, ${ }^{1}$ this covariate may have biased our results of the effects of childhood sexual abuse on characteristics of adult depression. Beyond the variables analysed in our meta-regressions and subgroup analyses, other factors such as sociodemographic status, additional adverse life events or parental psychiatric disorders could also be correlated with our independent and dependent variables and therefore distort effect sizes.

Finally, most primary studies used a cross-sectional design with currently depressed patients retrospectively reporting on childhood maltreatment. This approach leaves reports vulnerable to recall and mood effect. However, recent studies found this effect to be negligible and showed retrospective reports to be highly consistent with prospective designs. ${ }^{35-37}$

Future research is needed to clarify both the differential effects of childhood maltreatment subtypes in their influence on age at depression onset, course of illness and treatment response as well as the role of potential mediators (i.e. comorbid disorders) or confounding variables. Results on these issues may well enable a more effective clinical management of the considerable group of patients with depression who have a history of childhood maltreatment.

Janna Nelson, MSC, Anne Klumparendt, MSC, Department of Psychology, University of Münster and Christoph-Dornier-Foundation for Clinical Psychology, Münster; Philipp Doebler, PhD, Faculty of Statistics, Technical University Dortmund, Dortmund; Thomas Ehring, PhD, Department of Psychology, LMU Munich, Munich, Germany

Correspondence: Thomas Ehring, PhD, LMU Munich, Leopoldstr. 13, 80802 Munich, Germany. Email: thomas.ehring@Imu.de

First received 23 Dec 2015, final revision 23 Jun 2016, accepted 29 Jul 2016

\section{Funding}

J.N. and A.K. are recipients of a PhD scholarship from the Christoph-Dornier-Foundation for Clinical Psychology, Münster.

\section{References}

1 Andrade L, Caraveo-Anduaga JJ, Berglund P, Bijl RV, De Graaf R, Vollebergh W, et al. The epidemiology of major depressive episodes: results from the International Consortium of Psychiatric Epidemiology (ICPE) Surveys. Int J Methods Psychiatr Res 2003; 12: 3-21.

2 Murray CJL, Vos T, Lozano R, Naghavi M, Flaxman AD, Michaud C, et al Disability-adjusted life years (DALYS) for 291 diseases and injuries in 21 regions, 1990-2010: a systematic analysis for the Global Burden of Disease Study 2010. Lancet 2012; 380: 2197-223.

3 Sharpley CF, Bitsika V. Validity, reliability and prevalence of four 'clinical content' subtypes of depression. Behav Brain Res 2014; 259: 9-15.

4 Harald B, Gordon P. Meta-review of depressive subtyping models. J Affect Disord 2012; 139: 126-40.

5 Heun R, Kockler M, Papassotiropoulos A. Distinction of early- and late-onset depression in the elderly by their lifetime symptomatology. Int J Geriatr Psychiatry 2000; 15: 1138-42.

6 Carter GC, Cantrell RA, Zarotsky V, Haynes VS, Phillips G, Alatorre $\mathrm{Cl}$, et al. Comprehensive review of factors implicated in the heterogeneity of response in depression. Depress Anxiety 2012; 29: 340-54.

7 Ghaemi SN. Why antidepressants are not antidepressants: STEP-BD, STARD, and the return of neurotic depression. Bipolar Disord 2008; 10: 957-68.

8 Driscoll HC, Basinski J, Mulsant BH, Butters MA, Dew MA, Houck PR, et al. Late-onset major depression: clinical and treatment-response variability. Int J Geriatr Psychiatry 2005; 20: 661-7.

9 Arata CM, Langhinrichsen-Rohling J, Bowers D, O'Farrill-Swails L. Single versus multi-type maltreatment: An examination of the long-term effects of child abuse. J Aggress Maltreat Trauma 2005; 11: 29-52.

10 Bernet $\mathrm{CZ}$, Stein MB. Relationship of childhood maltreatment to the onset and course of major depression in adulthood. Depress Anxiety 1999; 9: 169-74.

11 Comijs HC, van Exel E, van der Mast RC, Paauw A, Voshaar RO, Stek ML. Childhood abuse in late-life depression. J Affect Disord 2013; 147: 241-6. 
12 Nanni V, Uher R, Danese A. Childhood maltreatment predicts unfavorable course of illness and treatment outcome in depression: a meta-analysis. Am J Psychiatry 2012; 169: 141-51.

13 Norman RE, Byambaa M, De R, Butchart A, Scott J, Vos T. The long-term health consequences of child physical abuse, emotional abuse, and neglect: a systematic review and meta-analysis. PLOS Med 2012; 9: 1-31.

14 Spertus IL, Yehuda R, Wong CM, Halligan S, Seremetis SV. Childhood emotional abuse and neglect as predictors of psychological and physical symptoms in women presenting to a primary care practice. Child Abuse Neg 2003; 27: 1247-58.

15 Jumper SA. A meta-analysis of the relationship of child sexual abuse to adult psychological adjustment. Child Abuse Negl 1995; 19: 715-28.

16 Neumann DA, Houskamp BM, Pollock VE, Briere J. The long-term sequelae of childhood sexual abuse in women: a meta-analytic review. Child Maltreat 1996; 1: 6-16.

17 Al-Modallal H, Peden A, Anderson D. Impact of physical abuse on adulthood depressive symptoms among women. Issues Ment Health Nurs 2008; 29 : 299-314.

18 Beitchman JH, Zucker KJ, Hood J E, daCosta GA, Akman D. A review of the short-term effects of child sexual abuse. Child Abuse Negl 1991; 15: 537-56.

19 Buist A. Childhood abuse, postpartum depression and parenting difficulties: a literature review of associations. Aust NZ J Psychiatry 1998; 32: 370-8.

20 Kendall-Tackett $\mathrm{K}$. The health effects of childhood abuse: four pathways by which abuse can influence health. Child Abuse Negl 2002; 26: 715-29.

21 Martin CMS, de Carvalho Tofoli SM, Von Werne Baes C, Juruena M. Analysis of the occurrence of early life stress in adult psychiatric patients: a systematic review. Psychol Neurosci 2011; 4: 219-27.

22 Tipton E. Small sample adjustments for robust variance estimation with meta-regression. Psychol Methods 2015; 20: 375-93.

23 Hedges LV, Tipton E, Johnson MC. Robust variance estimation in metaregression with dependent effect size estimates. Res Synth Methods 2010; 1: 39-65.

24 Fisher Z, Tipton E. Robumeta: Robust Variance Meta-Regression. R package version 1.3. R-project.org, 2014 (http://CRAN.R-project.org/ package $=$ robumeta)
25 Higgins JPT, Thompson SG. Quantifying heterogeneity in a meta-analysis Stat Med 2002; 21: 1539-58.

26 Rothstein HR, Sutton AJ, Borenstein M. Publication Bias in Meta-Analysis: Prevention, Assessment and Adjustments. John Wiley and Sons, 2005.

27 Egger M, Davey Smith G, Schneider M, Minder C. Bias in meta-analysis detected by a simple graphical test. BMJ 1997; 315: 629-34.

28 Sterne JAC, Egger M. Regression methods to detect publication and other bias in meta-analysis. In Publication bias in Meta-Analysis: Prevention, Assessment, and Adjustments (eds HR Rothstein, AJ Sutton, M Borenstein): 99-110. Wiley, 2005.

29 Duval S, Tweedie R. Trim and fill: a simple funnel plot-based method of testing and adjusting for publication bias in meta-analysis. Biometrics 2000; 56: 455-63.

30 Peters JL, Sutton AJ, Jones DR, Abrams KR, Rushton L. Comparison of two methods to detect publication bias in meta-analysis. JAMA 2006; 295: 676-80.

$31 \mathrm{Holm} \mathrm{S}$. A simple sequentially rejective multiple test procedure. Scand J Statist 1979; 6: 65-70.

32 Tipton E, Pustejovsky JE. Small-sample adjustments for tests of moderators and model fit using robust variance estimation in meta-regression. J Educ Behav Stat 2015; 40: 604-34.

33 Peyrot WJ, Middeldorp CM, Jansen R, Smit JH, de Geus EJ, Hottenga JJ, et al. Strong effects of environmental factors on prevalence and course of major depressive disorder are not moderated by 5 -HTTLPR polymorphisms in a large Dutch sample. J Affect Disord 2013; 146: 91-9.

34 Cohen J. Statistical Power Analysis for the Behavioral Sciences (2nd edn). Erlbaum, 1988.

35 Pinto R, Correia L, Maia Â. Assessing the reliability of retrospective reports of adverse childhood experiences among adolescents with documented childhood maltreatment. J Fam Violence 2014; 29: 431-8.

36 Fergusson DM, Horwood $\mathrm{L}$, Boden JM. Structural equation modeling of repeated retrospective reports of childhood maltreatment. Int J Methods Psychiatr Res 2011; 20: 93-104.

37 Scott KM, McLaughlin KA, Smith DAR, Ellis PM. Childhood maltreatment and DSM-IV adult mental disorders: Comparison of prospective and retrospective findings. Br J Psychiatry 2012; 200: 469-75. 